\title{
Oral anticoagulants reduce dementia risk in patients with $\mathrm{AF}$
}

In addition to an increased incidence of stroke, atrial fibrillation (AF) is also linked with an elevated risk of dementia. A retrospective registry study published in the European Heart Journal now reports that oral anticoagulation therapy can protect against dementia in patients with $\mathrm{AF}$.

Although anticoagulation agents are frequently used to protect against the formation of stroke-inducing large emboli in patients with $\mathrm{AF}$, their effectiveness in protecting against smaller emboli that might result in cognitive decline is less clear. Ideally, a randomized, placebo-controlled trial should be performed to answer this question, but as the study investigators point out, "such a study will never be done due to ethical reasons; it is not possible to treat AF patients at risk of stroke with placebo". Therefore, they performed a retrospective cohort study to compare the incidence of dementia in patients with AF from a Swedish register.

Among all the patients with $\mathrm{AF}$ included in the analysis, 241,160 (54.3\%) did not receive oral anticoagulant therapy, 190,570 (42.9\%) received warfarin, 12,916 (2.9\%) received non-vitamin $\mathrm{K}$ oral anticoagulants, and $199(0.04 \%)$ received phenprocuomon. The incidence of dementia in patients treated with anticoagulants was lower than in patients who did not receive anticoagulants (1.14 versus 1.78 per 100 patient-years; $P<0.001)$. Propensity score matching showed that patients receiving anticoagulants had a $29 \%$ lower risk of dementia than patients not receiving anticoagulants (HR 0.71, 95\% Cl 0.68-0.74).

"Early initiation of anticoagulant treatment in patients with AF could be of value to preserve cognitive function," conclude the investigators. Additional registry studies are needed to corroborate these findings.

Karina Huynh

ORIGINAL ARTICLE Friberg, L. \& Rosenqvist, M. Less dementia with oral anticoagulation in atrial fibrillation. Eur. HeartJ. http://dx.doi.org/10.1093/eurheartj/eh×579 (2017)

FURTHER READING Sabir, l. et al. Oral anticoagulants for Asian patients with atrial fibrillation. Nat. Rev. Cardiol.11, 290-303 (2014) 